
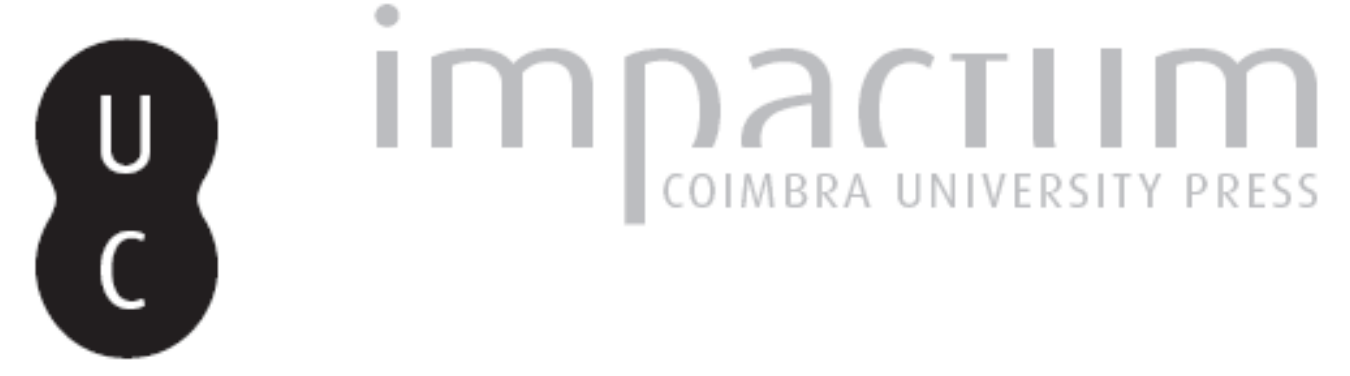

\title{
Vulnerabilidad de la región mediterránea peninsular a la hidrofobia de suelos
}

Autor(es): Ceballos Barbancho, Antonio

Publicado por: Associação Portuguesa de Riscos, Prevenção e Segurança

URL persistente:

URI:http://hdl.handle.net/10316.2/40054

DOI:

DOI:https://doi.org/10.14195/1647-7723_11_4

Accessed : $\quad$ 26-Apr-2023 15:06:46

A navegação consulta e descarregamento dos títulos inseridos nas Bibliotecas Digitais UC Digitalis, UC Pombalina e UC Impactum, pressupõem a aceitação plena e sem reservas dos Termos e Condições de Uso destas Bibliotecas Digitais, disponíveis em https://digitalis.uc.pt/pt-pt/termos.

Conforme exposto nos referidos Termos e Condições de Uso, o descarregamento de títulos de acesso restrito requer uma licença válida de autorização devendo o utilizador aceder ao(s) documento(s) a partir de um endereço de IP da instituição detentora da supramencionada licença.

Ao utilizador é apenas permitido o descarregamento para uso pessoal, pelo que o emprego do(s) título(s) descarregado(s) para outro fim, designadamente comercial, carece de autorização do respetivo autor ou editor da obra.

Na medida em que todas as obras da UC Digitalis se encontram protegidas pelo Código do Direito de Autor e Direitos Conexos e demais legislação aplicável, toda a cópia, parcial ou total, deste documento, nos casos em que é legalmente admitida, deverá conter ou fazer-se acompanhar por este aviso.

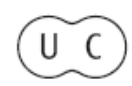




\title{
Vulnerabilidad de la región mediterránea peninsular a la hidrofobia de suelos
}

\author{
Antonio Ceballos Barbancho*
}

\begin{abstract}
Resumo:
A repelência à água ou hidrofobia é um processo hidrológico muito favorecido pelas características mediterrâneas de grande parte da Península Ibérica: estacionalidade das precipitações, "deficit" hídrico estival, vegetação emissora de substâncias hidrófobas, incêndios florestais, etc. As conclusões doúltimo relatório do IPCC (Intergovernmental Panel on Climatic Change) sugerem um provável aumento da frequência e intensidade da hidrofobia que agravará a actual fragilidade dos ccossistemas mediterrâneos perante processos de degradação ambiental. Esta situação convida a desenvolver linhas de investigação com o objectivo de avaliar o alcance destes processos.
\end{abstract}

Palavras chave:

Hidrofobia, degradação ambiental, ecossistemas mediterrâneos, Península Ibérica.

Résumé:

La répulsion à l'eau ou hydrophobie est un processus hydrologique très favorisé par les caractéristiques méditerranéennes d'une grande partie de la Péninsule Ibérique : saisonnalité des précipitations, déficit hydrique estival, végétation productrice de substances hydrophobes, incendies de forêt, etc. Les conclusions du dernier rapport de l'IPCC (Intergovernmental Panel on Climatic Change) suggère une probable augmentation de la fréquence et de l'intensité de l'hydrophobie qui aggravera l'actuelle fragilité des écosystèmes méditerranéens face aux processus de dégradation environnementale. Cette situation amène à développer des lignes de recherche ayant pour objectif l'évaluation de la portée de ces processus.

Mots clés:

Hydrophobie, dégradation environnementale, écosystème méditerranéen, Péninsule Ibérique.

\section{Abstract:}

Water repellence or hydrophobicity is a hydrological process that is favoured by the Mediterranean characteristics of a large part of Iberian Peninsula: intra-annual rainfall distribution, water deficit during the summer, vegetation that produces hydrophobic substances, forest fires, etc. The conclusions of the last report edited by the IPCC (Intergovernmental Panel on Climatic Change) suggest a probable increase in the frequency and intensity of hydrophobicity that will further worsen the present fragility of Mediterranean ecosystems in relation to degradation processes. This circumstance suggests the appropriateness of developing research projects aimed at evaluating the consequences of these processes. Key words:

Hydrophobocity, environmental degradation, Mediterranean ecosystems, Iberian Peninsula.

\section{¿Qué es la hidrofobia de suelos?}

La hidrofobia (soil hydrophobicity) de un suelo es el resultado del predominio de las fuerzas de cohesión entre las propias moléculas del agua sobre las fuerzas de atracción entre el suelo y el agua (adhesión). Puede definirse como una propiedad característica de ciertos suelos, la cual consiste en la reducción de la afinidad de ese suelo al agua. Por lo tanto, hidrofobia es sinónimo de repelencia al agua (water repellency), proceso que puede tener notables consecuencias hidrológicas, geomorfológicas, ecológicas y económicas según su grado de intensidad (DOERR et al., 2000).

\footnotetext{
* Departamento de Geografía. Universidad de Salamanca. C/ Cervantes, s/ n. 37.002 (España). ceballos@usal.es
}

El primer trabajo que aborda de manera específica la investigación de la hidrofobia es el publicado en 1932 por PRESCOTT \& PIPER, quienes observaron el proceso de repelencia al agua en una clásica formación arbustivà regresiva típica (mallee), bajo clima mediterráneo, en el continente australiano. En la década de los 40 destaca el trabajo de JAMISON (1947) sobre la repelencia al agua en las plantaciones de cítricos en California. No obstante, como señala DeBANO (2000), no es hasta finales de los 60 del pasado siglo cuandocomienzan a proliferar los estudios sobre este tema, especialmente en Estados Unidos y Australia, con motivo de la Conferencia de Riverside en California (DeBANO \& LETEY, 1969) en donde se presentaron un total de 31 trabajos referentes a diversos aspectos relacionados con la hidrofobia de suelos. 
A raíz del impulso de la década anterior, en los setenta se publicaron varios trabajos que aportaron una valiosa información para consolidar el conocimiento sobre la repelencia al agua o hidrofobia en numerosas áreas a escala mundial: Australia (ROBERTS \& CARBON, 1971), Egipto (BISHAY \& BAKHATI, 1976), Estados Unidos (MEEUWING, 1971; SCHOLL, 1975), la India (DAS \& DAS, 1972), Japón (NAKAYA et al., 1977), Nepal (CHAKRABARTI, 1971), Mali (RIETVELD, 1978) y Nueva Zelanda (JOHN, 1978). Los contenidos de estos trabajos inciden esencialmente en evaluar las consecuencias de la hidrofobia en el funcionamiento y gestión tanto de ecosistemas como de agrosistemas. Uno de los temas más desarrollados en esta década fue la influencia de los incendios forestales sobre la ocurrencia e intensidad de la hidrofobia, con numerosos trabajos experimentales llevados a cabo en Estados Unidos, que aparecen resumidos y sintetizados en la revisión publicada por DeBANO en 1981.

En la década de los ochenta DeBANO (2000) contabiliza alrededor de 150 publicaciones sobre hidrofobia de suelos en diversas revistas científicas. Este elevado número de aportaciones se puede dividir en aquellas publicaciones destinadas a estudiar las relaciones entre hidrofobia y fracciones texturales (por ejemploMcGUIE \& POSNER, 1980), hidrofobia y la existencia de zonas secas en campos de golf (por ejemplo TAYLOR \& BLAKE, 1982), la relación entre humus, materia orgánica parcialmente descompuesta e hidrofobia en ecosistemas forestales (GRELEWICZ \& PLICHTA, 1985), la influencia de la hidrofobia sobre la respuesta hidrológica a escala de cuenca (BURCH et al., 1989), entre otras.

La década de los 90 del siglo pasado puede calificarse como la de la "madurez" de las investigaciones sobre hidrofobia (DeBANO, 2000), con una abundante producción científica diseminada en los campos de la edafología, agronomía, geomorfología, geoquímica e hidrología (DOERR et al., 2000). Entre las líneas de investigación emprendidas en estos años, ha sido especialmente prolífica la labor desarrollada por el grupo de Coen Ritsema y Louis Dekker (Wageningen Agricultural University), en varias áreas experimentales, combinando trabajos de campo y laboratorio, y prestando una especial atención al campo de la modelización (DEKKER, 1998; RITSEMA, 1998). Otro grupo de investigación notable ha sido el encabezado por Stefan Doerr (Department of Geography, University of Swansea) con varias investigaciones desarrolladas en Portugal, en colaboración con el Departamento de Ambiente e Ordenamento de la Universidade de Aveiro. Fruto de esta colaboración ha sido un interesante conjunto de publicaciones que recogen los principales resultados, así como una serie de aportaciones metodológicas sumamente útiles para las mediciones de campo
(DOERR, 1998; DOERR et al., 1996; DOERR et al., 1998; DOERR \& THOMAS, 2000). En el ámbito de las cuencas experimentales, merece una especial mención la labor de David Scott en Sudáfrica (SCOTT, 1993; 2000; SCOTT et al., 1998), el cual ahora desarrolla su investigación en la Columbia Británica.

\section{Factores que determinan la ocurrencia e intensidad de la hidrofobia}

\section{Fuentes de emisión de sustancias hidrófobas}

La aparición de la hidrofobia está directamente asociada a la precipitación de una serie de sustancias, generalmente orgánicas, que se adhieren a las partículas del suelo, a través de diversos procesos. Una primera fuente se debe a las partículas microscópicas de materia orgánica procedentes de la descomposición de una serie de plantas que se adhieren a la fracción mineral del suelo, sellando los espacios intersticiales y formando una película hidrófoba (FRANCO et al., 1994). En relación con la vegetación, una segunda fuente sería la exudación de sustancias hidrófobas a través de las raíces de algunas especies de árboles, siendo un ejemplo muy estudiado los casos del Eucalyptus globulus y el Pinus pinaster (FERREIRA, 1996; DOERR et al., 1996). En tercer lugar, el lavado de ciertas ceras y resinas procedentes de la masa foliar de determinadas especies vegetales puedederivar en la formación de una película hidrófoba en el horizonte superficial del suelo. A modo de ejemplo pueden citarse los casos de especies netamente mediterráneas como Cistus monspelliensis (GIOVANNINI et al., 1987), Quercus turbinella (HOLZHEY, 1969), Quercus suber (SEVINK et al., 1989) o Quercus ilex subsp. ballota (CERDÀ et al., 1998). Finalmente, las hifas de algunas especies de hongos, como los basidiomicetos, pueden exudar sustancias hidrófobas según han documentado entre otros WILLKINSON \& MILLER (1978), EMERSON(1993) y YORK(1998).

Respecto a la naturaleza química de la sustancia impregnante, la literatura manifiesta una apreciable falta de información (DOERR et al., 2000), citándose como sustancias hidrófobas los hidrocarbonos alifáticos C-H(CAPRIEL, 1997; MORALet al., 2002) y sustancias polares de estructura anfifílica como una cadena de átomos de carbono que no se disuelven en el agua debido a su especial orientación (DOERR et al., 2000).

\section{El factor climático}

Todas las zonas, en donde se ha detectado la presencia de suelos hidrófobos, se caracterizan por una significativa ausencia de precipitaciones durante un determinado 
período de tiempo, circunstancia fundamental para explicar la formación, estabilidad y actuación de la película hidrófoba (FERREIRA, 1996). Al respecto, trabajos recientes como el de JARAMILLO et al. (2000) matizan la hipótesis, generalmente aceptada, que defiende que la hidrofobia es común en climas secos y muy excepcional en el caso de climas húmedos. Estos autores argumentan, de forma novedosa, que el efecto real del clima sobre la hidrofobia es limitado y que el verdadero impacto del clima parece estar más relacionado con su capacidad de producir materia orgánica portadora de sustancias hidrófobas. Un clima extremadamente seco se caracterizará por presentar unos ratios muy bajos de producción de materia orgánica, mientras que un clima húmedo es capaz de generar un volumen de materia orgánica suficiente para emitir sustancias hidrófobas al medio y si, además, presenta algunos períodos de ausencia de lluvia, puede constituir un ambiente potencial para la ocurrencia de la hidrofobia. Un buen ejemplo lo encontramos en los trabajos desarrollados en medios húmedos y subhúmedos por JUNGERIUS \& DEJONG (1989), DEKKER (1998) y RITSEMA (1998).

Con independencia del régimen pluviométrico discutido en el párrafo anterior, se puede definir la hidrofobia como un proceso temporalmente discontinuo. Su correspondenciacon períodos sin precipitación (rachas secas) ha sido demostrada por los trabajos de CROCKFORD et al. (1991) y WALSH et al. (1994), que describen como durante lluvias continuadas, que provocan el relleno del medio poroso con agua y la recuperación de los contenidos de humedad del suelo, la hidrofobia desaparece para reaparecer una vez que las lluvias cesan y los suelos se secan. El restablecimiento de la hidrofobia se debe o bien a nuevas entradas de sustancias hidrófobas en el sistema edáfico o bien a la reorientación de los átomos que componen las cadenas anfifílicas descritas con anterioridad (DOERR \& THOMAS, 2000). Son escasísimos los estudios que señalan un umbral de humedad a partir del cual la hidrofobia desaparece. Por ejemploCEBALLOS et al. (1999) señalan un umbral del $14 \%$ vol. en la región central de Portugal, con una precipitación media anual por encima de los $1.000 \mathrm{~mm}$ pero con el registro de al menos dos meses secos al año. DOERR et al. (2000) apuntan la dependencia que este umbral puede tener de la propia fracción textural del suelo.

\section{El factor edáfico}

Aparte del factor climático, el suelo tiene que presentar una serie de propiedades físicas que le hagan susceptible a la formación de una película repelente al agua (WALLIS \& HORNE, 1992). Entre las mismas destaca la textura, ya que suelos con texturas gruesas, caracterizados por un contenido de arcilla inferior al $5 \%$, favorecen la adhesión de las sustancias hidrófobas a las partículas minerales (MOORE \& BLACKWELL, 1998) y el sellamiento efectivo de los espacios intersticiales del suelo. Al respecto, el trabajo de HARPER \& GILKES (1994) incluye una escala de suelos que relaciona textura y grado de repelencia al agua en donde puede apreciarse que los suelos con mayor grado de repelencia son aquellos con menores contenidos de arcilla. Esta relación se explica porque los suelos arenosos presentan una menor superficie específica que los suelos limosos o arcillosos, circunstancia que facilita la adhesión de las sustancias hidrófobas, siempre en una cantidad limitada en el medio edáfico. No obstante, en suelos muy ricos en arcillas también se ha detectado hidrofobia como consecuencia de la formación de micro-agregados que mostraron un comportamiento similar a la de los suelos con fracción arenosa predominante (CROCKFORD etal., 1991;BISDOMetal., 1993; DEKKER \& RITSEMA, 1996). Finalmente, en consonancia con la explicación previa, conforme mayor sea la emisión de sustancias hidrófobas y su concentración en el suelo, menor será la relación entre ocurrencia e intensidad de la hidrofobia y el predominio de texturas groseras.

En relación con el contenido de materia orgánica, autores como WALLIS et al. (1990) y McKISSOCK et al. (1998) encontraron una relación positiva entre volumen de materia orgánica y grado de hidrofobia, aunque los resultados de otros trabajos no expresan una relación clara entre ambas variables (DeBANO, 1991; WALLIS et al. 1993; CEBALLOS et al., 1999), debido a que no existe una proporcionalidad entre cantidad de materia orgánica y volumen de sustancias hidrófobas en el suelo.

\section{Los incendios forestales y fuegos controlados}

Según ha documentado Leonard DeBano en numerosas publicaciones (i.e.: DeBANO, 1981; DeBANO et al., 1976; 1998), el fuego, o bien puede provocar la aparición de la hidrofobia en suelos previamente hidrófilos o bien atenuar o destruir las capas hidrófobas del suelo tras el desarrollo del incendio, ya que la influencia del fuego sobre la hidrofobia está estrechamente relacionada con la temperatura alcanzada durante la combustión. DeBANO et al. (1976) apuntan que la hidrofobia se intensifica con temperaturas comprendidas entre los 175 y $200^{\circ} \mathrm{C}$, mientras que a partir de $\operatorname{los} 270-300^{\circ} \mathrm{C}$ la hidrofobia desaparece debido a la destrucción de las sustancias que la provocan. No obstante, en la mayoría de los casos estudiados (DeBANO, 1981), el proceso de combustión durante el incendio suele provocar la migración de las sustancias hidrófobas a lo largo del marcado gradiente térmico 
provocado por la escasa conductividad del suelo que se traduce en la formación de un potente horizonte hidrófobo a una profundidad que dependerá de la temperatura alcanzada en la superficie del suelo.

\section{Susceptibilidad de la región mediterránea a padecer la hidrofobia de suelos}

La característica configuradora del clima mediterráneo es la coincidencia del mínimo pluviométrico anual con la máxima evapotranspiración, debido a las altas temperaturas. Ello provoca un decrecimiento ostensible de la humedad del suelo y sobre todo la estabilidad, durante un período de tiempo apreciable, de las sustancias responsables de la formación de la película hidrófoba. Más de las dos terceras partes de la Península Ibérica pertenecen al dominio del clima mediterráneo, caracterizado por la existencia de al menos dos meses secos al año, con un ombroclima que oscila desde semiárido (precipitaciones anuales inferiores a $350 \mathrm{~mm}$ ), en el cuadrante suroriental peninsular, hasta hiperhúmedo (precipitaciones anuales superiores a $1.600 \mathrm{~mm}$ ), en el sector occidental de las Sierras Béticas. Considerando el concepto de racha seca (período con un número de días consecutivos sin precipitación apreciable), podemos analizar la importancia de la ausencia de precipitaciones sobre la ocurrencia de hidrofobia en la Península Ibérica. A partir del trabajo de MARTÍN-VIDE \& GÓMEZ (1999), si adoptamos 0, 1 mm como umbral de precipitación que diferencie entre día seco y día lluvioso, la duración media de las rachas secas en la mitad de la Península Ibérica es 7 días. Utilizando como umbral de lluvia $10 \mathrm{~mm}$, debido a la especial incidencia de lluvias de esta cuantía sobre el frente de humedad del suelo (CEBALLOS et al., 2002a; 2004), en la dos terceras partes de la Península Ibérica la duración media de las rachas secas es superior a 25 días.

Por otro lado, la región mediterránea es uno de los espacios más sensibles a la mayor frecuencia en el futuro de eventos climáticos extremos, con una creciente concentración de las precipitaciones en invierno y sequías más prolongadas en el verano (IPCC, 2001). El trabajo de AYALA-CARCEDO \& IGLESIAS (2000) pronostica, para una gran parte del territorio peninsular, un aumento de la temperatura media anual de $2,5^{\circ} \mathrm{C}$, un decrecimiento del $8 \%$ de la precipitación media anual respecto a los valores actuales e, integrando ambas variables, un aumento medio de la evaporación del $22 \%$ para el año 2060 . Este escenario puede redundar en una más acusada estabilidad temporal de la hidrofobia en ciertos ecosistemas.

La región mediterránea es una de las zonas del Planeta más proclives a sufrir incendios forestales (REBELO, 2003), debido a la interacción positiva de los factores naturales y antrópicos, con una tendencia creciente en las últimas décadas. A modo de ejemplo, ALEXANDRIAN et al. (1999) apuntan que en Grecia se ha pasado de 12.000 ha/año en 1970 a 39.000 en la actualidad; en Italia de 43.000 a 118.000 y en España de 50.000 a 208.000. Según los informes publicados por la Comisión Europea (EFFIS-European Forest Fires Information System-) en la Península Ibérica ardieron más de 450.000 has (el $84 \%$ de esta superficie quemada pertenece a Portugal) durante el verano de 2003. Esta circunstancia, de cara a la ocurrencia de la hidrofobia, es muy significativa, ya que los suelos, generalmente forestales, de las superficies incendiadas suelen tener un contenido de materia orgánica superior al $2 \%$ (por ejemplo, en el caso de los incendios del año 2003, una parte considerable de las superficies afectadas estaban cubiertas por repoblaciones forestales de Pinus pinaster y Eucalyptus globulus, especies claramente emisoras de sustancias hidrófobas). DeBANO (1991) demuestra que el calentamiento de un suelo previamente hidrófilo que contenga más de un 2-3\% de materia orgánica puede inducir a la aparición de la hidrofobia.

Finalmente, para evaluar la vulnerabilidad de la región mediterránea a sufrir la hidrofobia de suelos habría que destacar que un importante grupo de las especies vegetales emisoras de sustancias hidrófobas son mediterráneas, muchas de las cuales forman parte de la flora ibérica (tabla 1):

\begin{tabular}{|cc|cc|}
\hline Nombre de la especie & Referencia bibliográfica & Nombre de la especie & Referencia bibliográfica \\
\hline Agrostis sp. & Wilkinson \& Miller (1978) & Quercus ilex subsp. ballota & Cerdà et al. (1998) \\
Calluna vulgaris & Mallik \& Rahmann (1985) & Pinus halepensis & Mataix-Solera \& Doerr (2004) \\
Cistus monspelliensis & Giovannini et al. (1987) & Pinus nigra & Gerke et al. (2001) \\
Chamaespartium tridentatum & Richardson \& Hole (1978) & Pinus pinaster & Shakesby et al. (1993) \\
Erica arborea & Giovannini et al. (1987) & Pinus pinea & Moral et al. (2002) \\
Erica cinerea & Ceballos et al. (1999) & Pinus radiata & Scott \& Van Wyk (1990) \\
Erica umbellata & Ceballos et al. (1999) & Populus tremula & Reeder \& Juergensen (1979) \\
Eucalyptus globulus & Doerr et al. (1996) & Retama sphaerocarpa & Contreras \& Solé (2003) \\
Quercus suber & Sevink et al. (1989) & Ulex europaeus & Soto et al. (1994) \\
Vaccinizm sp. & Richardson \& Hole (1978) & & \\
\hline
\end{tabular}

Tabla 1 - Especies de plantas asociadas a la emisión de sustancias hidrófobas presentes en la Península lbérica 


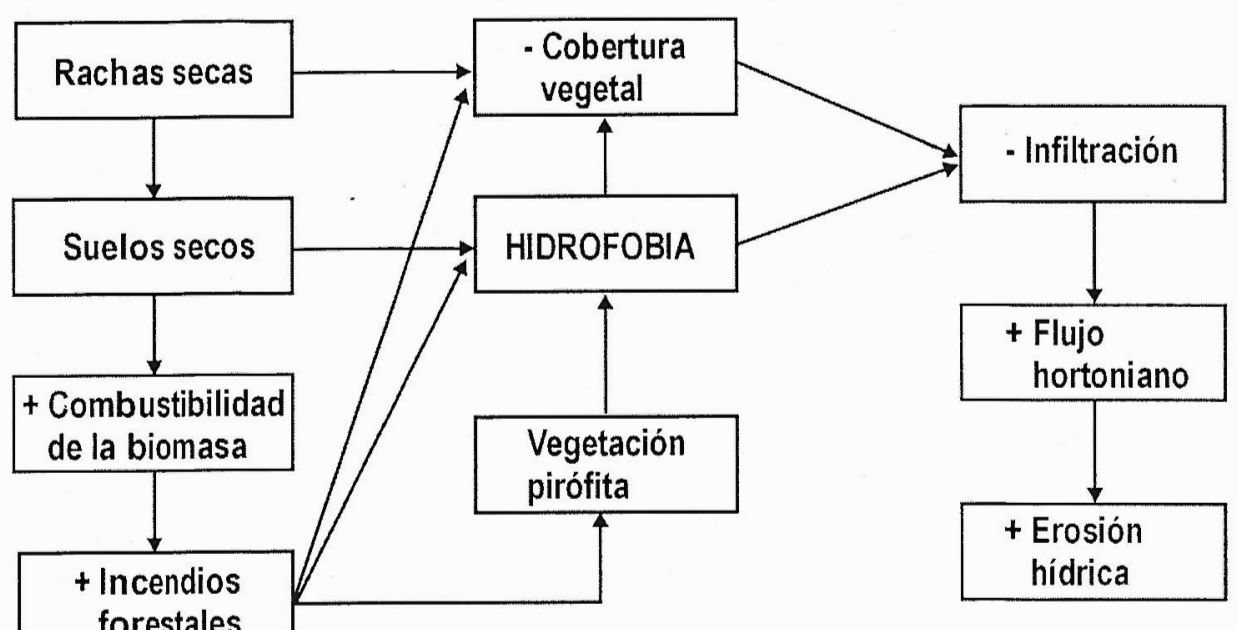

Figura 1 - Relación entre dinámica natural de los ecosistemas mediterráneos, ocurrencia de hidrofobia y degradación del medio.

Este conjunto de especies actúa como una considerable fuente de sustancias hidrófobas a través de diferentes vías: exudación de los sistemas radiculares, lavado de ceras y resinas procedentes de las hojas, descomposición de restos vegetales, etc. Las superficies ocupadas por las formaciones vegetales regresivas típicas de los ecosistemas mediterráneos como la garriga, la maquia y el chaparral han sido consideradas como enclaves susceptibles para la ocurrencia de procesos intensos de hidroforbia (SCHOLL, 1975; GIOVANNINI et al, 1987; DeBANO, 1991). Algunos autores haninterpretado este comportamiento de algunas especies de plantas en términos de dinámica y adaptación ecológica -alelopatía-(SCOTT, 1992; MOORE \& BLACKWELL, 1998), bien para inhibir la germinación de especies competidoras, o bien para favorecer la infiltración del agua a horizontes más profundos, vía flujo preferencial a través de macroporos, para minimizar las pérdidas por evaporación y disponer de una reserva en el nivel radicular del perfil edáfico.

Como muestra la figura 1, a modo de conclusión puede afirmarse que la interacción de todos estos factores favorece la ocurrencia e intensidad de la hidrofobia, incrementando la actual fragilidad de los ecosistemas mediterráneos ante procesos de degradación ambiental.

\section{Riesgos derivados de la ocurrencia de hidrofobia de suelos}

Las consecuencias de la hidrofobia sobre la dinámica de cualquier ecosistema son numerosas y complejas, dependiendo de su grado de intensidad.

En relación con la dinámica y disponibilidad de agua, la hidrofobia reduce la capacidad de infiltración del suelo aumentando la producción de escorrentía superficial y acortando los tiempos de respuesta (picos del hidrograma) ante determinadas precipitaciones. A escala de pedón $\left(0,24 \mathrm{~m}^{2}\right)$, mediante experimentos de simulación de lluvia, CERDÀ et al. (1998) y CEBALLOS et al. (2002b) revelan que, a pesar del escaso contenido de humedad del suelo y de la importancia del espacio poroso libre de agua, en suelos afectados por la hidrofobia el tiempode respuesta es muy corto, con una tasa de infiltración inicial muy baja (figura 2). Estos trabajos confirman las conclusiones de la investigación previa de WALLIS et al. (1990) que señalaban que la capacidad de infiltración en un suelo arenoso repelente fue seis veces menor respecto a un suelo no repelente adyacente y más húmedo. A escala de parcelas experimentales $(8 \times 2 \mathrm{~m})$, FERREIRA et al. (1998) demuestran la efectividad de la hidrofobia en áreas forestales de pinos y eucaliptus. Finalmente, a pesar de la discontinuidad espacial de la hidrofobia, trabajos como los de BURCH et al. (1989) y SCOTT et al. (1998) reflejan el efecto de la hidrofobia sobre el funcionamiento hidrogeomorfológico de pequeñas

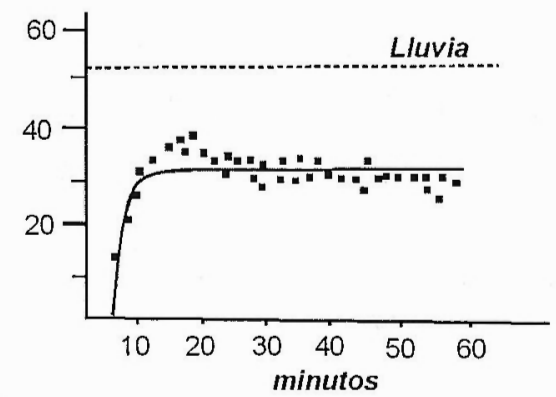

Figura 2 - Hidrograma correspondiente a una superficie hidrófoba en una dehesa en Extremadura. Experimento de simulación de lluvia aplicando una intensidad constante de $53 \mathrm{~mm} / \mathrm{h}$. Gráfico basado en CERDÀ et al. (1998) y CEBALLOS et al. (2002b). 
cuencas experimentales. En la mayoría de los casos, debido a la concentración de las sustancias hidrófobas en el horizonte superficial del suelo, el modelo de escorrentía generado responderá al tipo superficial hortoniano. No obstante, una localización de la capa hidrófoba a varios centímetros de profundidad desde la superficie puede provocar flujo subsuperficial a través del medio poroso del suelo y flujo superficial por saturación de los primeros centímetros (DOERR et al., 2000).

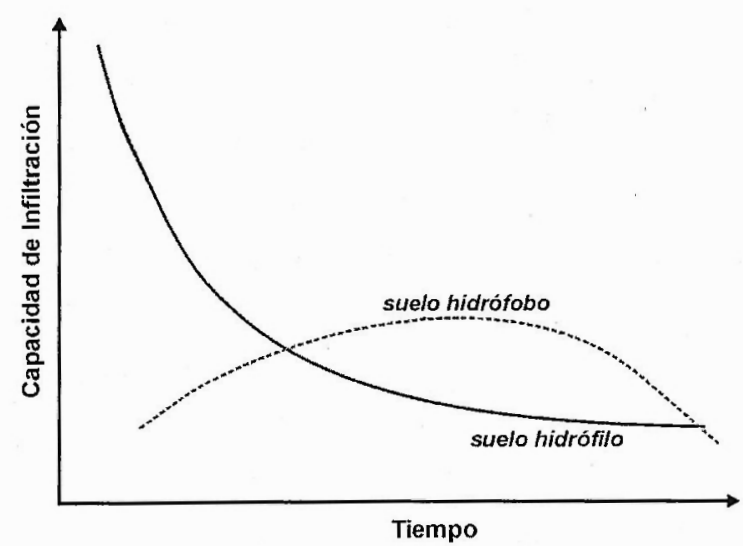

Figura 3: Curvas de infiltración habituales en suelos hidrófobos e hidrófilos respectivamente.

La modificación de la curva habitual de infiltración (figura 3), provocada por la hidrofobia, dificulta enormemente la introducción del proceso de infiltración de un suelo hidrófobo en un modelo hidrológico mediante una ecuación. Así, un factor de incertidumbre es el valor de humedad a partir del cual la hidrofobia desaparece y la tendencia de la infiltración cambia sustancialmente.

Junto al aumento del volumen de escorrentía superficial, otra implicación hidrológica muy notable de la hidrofobia (y aparentemente contradictoria con la anterior) es el favorecer los flujos a través de macroporos, con la producción de procesos de percolación, dando lugar a frentes de humedad heterogéneos, aunque el suelo no esté saturado (WILSON et al., 1990; HENDRICX et al., 1993). El origen de los macroporos suele estar relacionado con la existencia de grietas en el suelo, fauna edáfica, discontinuidades texturales, raíces secas, etc. En la figura 4 se aprecia claramente el flujo de agua a través de macroporos, en un perfil edáfico, analizando la evolución de la humedad del suelo durante un experimento de simulación de lluvia con sondas TDR (Time Domain Reflectometry). Mientras la reacción de las sondas situadas a 5 y $10 \mathrm{~cm}$ de profundidad es apenas perceptible, la reacción de la sonda situada a $15 \mathrm{~cm}$ es muy notable ante la llegada de agua directa a través de macroporos cubiertos con sustancias hidrófobas. El flujo de agua a través de macroporos inducido por la hidrofobia, puede repercutir en la pérdida de solutos en superficie que alcanzan rápidamente el nivel freático con riesgos de contaminación de las aguas subterráneas (HENDRICX et al., 1993).

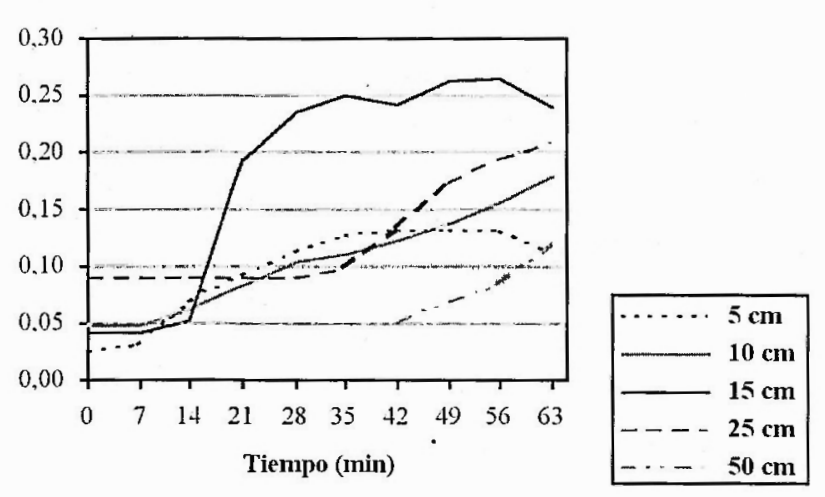

Figura 4 - Evolución de la humedad del suelo en un luvisol, afectado por hidrofobia en los primeros centímetros del perfil (CEBALLOS et al., 2002c).

En determinados ambientes se ha detectado un incremento de la erosión, con la formación de regueros y cárcavas, relacionados con la acción de escorrentías superficiales, en ciertos tipos de suelo que de no ser frecuentemente hidrófobos no hubieran tenidoeste tipode modelado(MOORE\& BLACKWELL, 1998). De igual manera, la aparición de un horizonte hidrófoboen profundidad, y los procesos de escorrentía subsuperficial derivados de ello, pueden provocar la formación de túneles (piping) susceptibles de ceder con posterioridad transformándoseen cárcavas (DOERR et al., 2000). La mayoría de los casos estudiados sobre la relación èntre erosión e hidrofobia corresponden azonas afectadas por incendios forestales. Esta circunstancia dificulta enormemente aislar el peso específico de la hidrofobia sobre el incremento de la pérdida de suelo debido a que otros factores derivados del propio incendio (desaparición de la cobertura vegetal del suelo, pérdida de materia orgánica, destrucción de agregados, etc) también inciden positivamente en dicho proceso. No obstante, los estudios realizados en Portugal por SHAKESBY et al. $(1994,1996)$ y WALSH et al. (1994) apuntan a la hidrofobia como el principal factor para explicar el incremento de la erosión medida en parcelas experimentales, especialmente durante eventos tormentosos precedidos de un período sin precipitaciones apreciables. Finalmente, SHAKESBY et al. (2000) sugieren que el impacto de 
la hidrofobia sobre la pérdida de suelo va a estar directamente relacionado con la continuidad espacial de esta: Si ésta es continua, permitirá la paulatina concentración de la escorrentía superficial, un incremento de su energía y, por tanto, de su capacidad de transporte de sedimentos.

Otras consecuencias de especial relevancia son, por un lado, que la inhibición de la infiltración da lugar a la existencia de zonas secas en el perfil del suelo (especialmente en las áreas de influencia de los sistemas radiculares de las plantas), que incrementan notablemente el estrés hídrico vegetal (HOUSE, 1991; MORAL et al., 2002), y, por otro, que la intensificación de la erosión hídrica puede derivar en un empobrecimiento de la fertilidad de los propios suelos agrícolas, con la pérdida de nutrientes y de la matriz mineral fina (MOORE \& BLACKWELL, 1998).

\section{La investigación de la hidrofobia en la Península Ibérica}

Si bien en el caso portugués la investigación de la hidrofobia ha sido en gran parte dirigida por la colaboración entre las universidades de Aveiro y Swansea, en el caso español, los estudios son más dispersos y puntuales. Asumiendo el riesgo de dejar en el tintero la referencia de algún trabajo notable, la siguiente síntesis puede ser útil para esbozar una idea general de la investigación de la hidrofobia en la Península Ibérica:

SEVINK et al. (1989) realizaron una serie de experimentos de simulación de lluvia que demostraron el efecto de la hidrofobia en suelos ricos en humus tipo mar, en alcornocales ( $Q$. suber) del noreste de España con cortejo florístico de ericáceas (brezos y madroños). En la misma zona IMESON et al. (1992), en un estudio sobre la incidencia de los incendios forestales sobre la respuesta hidrológica de los suelos, asociaron la discontinuidad espacial de los procesos de escorrentía superficial e infiltración a la ocurrencia de hidrofobia.

El trabajo de DOERR et al. (1996) concluye que en las plantaciones forestales de Pinus pinaster y Eucalyptus globulus en el norte de Portugal no se aprecia una relación entre la intensidad de hidrofobia y la ocurrencia previa de incendios forestales. Igualmente, los eucaliptus parecen ser más hidrófobos que los pinos. El trabajo de DOERR \& THOMAS (2000) aborda la relación entre humedad del suelo e hidrofobia, matizando la simplicidad de la relación habitualmente divulgada en la literatura.

DOERR et al. (1998) destacan los altos valores de hidrofobia medidos in situ en plantaciones forestales de Pinus pinaster y Eucalyptus globulus en Portugal, así como su homogeneidad espacial debido a la regularidad en el modelo de distribución de los árboles.

CERDÀ et al. (1998) y CEBALLOS et al. (2002b), mediante experimentos de simulación de lluvia, detectaron la aparición de hidrofobia en las dehesas de Extremadura (oeste de España), concretamente en las zonas situadas bajo la proyección de las copas de $Q$. ilex subsp. ballota, debido a la existencia de ceras procedentes, con toda probabilidad, del lavado de las hojas de las encinas. Los resultados de estos trabajos demostraron la relación entre hidrofobia, disminución de la infiltración, aumento de la escorrentía superficial e incremento de las tasas de erosión a escala de pedón.

En eláreamontañosacentral de Portugal,CEBALLOS et al. (1999) demuestran que las zonas sometidas a una práctica continuada de fuego controlado no experimentan hidrofobia al inhibir el desarrollo de especies arbustivas hidrófobas propias de una fase más avanzada en el proceso de sucesión vegetal. Al mismo tiempo, el fuego controlado de baja intensidad no afecta a las principales propiedades físicas e hidrológicas del suelo (porosidad, textura, materia orgánica, retención hídrica). Este trabajo sugiere la importancia cualitativa más que cuantitativa de la materia orgánica para la ocurrencia de la hidrofobia. Al igual que sugieren SEVINK et al. (1989), aunque la hidrofobia en zonas ricas en humus y protegidas por la vegetación favorezca notablemente el proceso de escorrentía, sin embargo el efecto erosivo de la misma es pequeño debido al efecto pantalla de la vegetación, la irregularidad de la superficie del terreno y la fijación de los sistemas radiculares.

El trabajo de FERREIRA et al. (2000), en la cuenca del río Águeda en Portugal, estudia la incidencia de la hidrofobia en áreas repobladas con Pinus pinaster y Eucalyptus globulus, en pequeñas cuencas experimentales, mostrando su efecto no sólo en el registro de un apreciable coeficiente de escorrentía, sino también en las características de los picos de los hidrogramas.

INGELMO et al. (2000) han realizado una serie de experiencias de laboratorio para confirmar la validez de los métodos habituales (WDPT y MED) de medición de la hidrofobia en una tipología amplia de suelos en la Comunidad Valenciana.

MORAL et al. (2001;2002) apuntan la existencia de hidrofobia en los suelos arenosos del manto eólico cuaternario del Parque Nacional de Doñana en el SO peninsular, en zonas con abundancia de pino piñonero $(P$. pinea). Además estos trabajos han incidido en el análisis de la relación entre contenido de materia orgánica en el suelo e intensidad de su repelencia al agua.

CONTRERAS \& SOLÉ (2003), han estudiado la hidrofobịa en suelos del SE peninsular asociada a Retama sphaerocarpa y a la localización puntual de 
letrinas de conejos (Oryctolagus cuniculus), siendo uno de los primeros trabajos que relacionan la ocurrencia espacial de la hidrofobia con restos orgánicos de especies animales. Este trabajo destaca la importancia de la vegetación, textura del suelo (suelos arenosofrancos) y las letrinas de los conejos en la aparición de la hidrofobia.

Finalmente, MATAIX-SOLERA \& DOERR (2004) han observado hidrofobia en el sureste peninsular (Alicante), en zonas afectadas por Pinus halepensis, a través de un muestreo puntual por medio de transectos. A pesar de que en la mayoría de los casos estudiados la hidrofobia se ha detectado en sustratos ácidos, en el presente trabajo se aprecia en suelos alcalinos. Este trabajo profundiza en la relación entre hidrofobia y estabilidad de agregados.

\section{Conclusiones}

La hidrofobia de suelos es un proceso netamente hidrológico característico de ciertas formaciones edáficas que muestran una clara repelencia al agua, inhibiendo la infiltración a través de la matriz del suelo. Su ocurrencia e intensidad está directamente relacionada con la confluencia de un conjunto de factores entre los que destacan la existencia de fuentes transmisoras de sustancias hidrófobas al medio (i.e. vegetación) y la persistencia de rachas secas o períodos $\sin$ lluvias que promueva la estabilidad de las sustancias hidrófobas en el suelo. Aunque los resultados de investigaciones previas sobre el tema muestran algunas contradicciones, determinadas propiedades edáficas, como fracciones texturales gruesas y una cierta proporción de materia orgánica, junto a la ocurrencia de incendios forestales, pueden intensificar el grado de repelencia al agua de un suelo.

Más de dos tercios de la superficie de la Península Ibérica tienen un marcado carácter mediterráneo (irregularidad de las precipitaciones, déficit hídrico estival, vegetación esclerófila y perennifolia) que agudiza la vulnerabilidad de este territorio ante determinados riesgos ambientales en el actual contexto de cambio global. El último informe del Panel Intergubernamental de Expertos sobre Cambio Climático (IPCC, 2001) predice, en el caso de la región mediterránea y a lo largo del presente siglo, un aumento de las temperaturas superior a $2,5^{\circ} \mathrm{C}$ y una mayor irregularidad de las precipitaciones con un decrecimiento de las mismas, entre un 5 y un $20 \%$, durante la estación estival respecto a la media actual. Estas circunstancias realimentarán la frecuencia e intensidad de olas de calor, incendios forestales, los problemas de disponibilidad de agua, crecidas hidrológicas, tasas de erosión y expansión de vegetación xerófila de carácter arbustivo. Sin duda, este nuevo escenario favorece la ocurrencia e intensidad de la hidrofobia que se traducirá en mayores coeficientes de escorrentía, una menor capacidad de almacenamiento y retención de agua en los ecosistemas y una mayor degradación de los recursos edáficos.

Durante la década de los 90 del siglo pasado se han realizado algunos estudios sobre la incidencia de la hidrofobia en la Península Ibérica, destacando por su número y calidad los correspondientes a la mitad septentrional de Portugal en superficies forestales ocupadas por Pinus pinaster y Eucalyptus globulus. En relación con los futuros escenarios ambientales, que algunas investigaciones sugieren para el ámbito mediterráneo peninsular, sería muy conveniente el desarrollo de líneas de trabajo que profundicen en la relación entre hidrofobia y especies características de las diversas etapas seriales de vegetación y en un mayor conocimiento de la retroalimentación positiva de la actual fragilidad de los ecosistemas mediterráneos por parte de la hidrofobia de suelos. Ello permitirá el diseño de estrategias que mitiguen el problema allí donde fuera necesario.

\section{Bibliografía}

ALEXANDRIAN, D., ESNAULT, F. \& CALABRI, G. (1999) "Forest fires in Mediterranean area". Unasylva, 50: 35-41.

AYALA-CARCEDO, F.J. \& IGLESIAS, A. (2000) - "Impactos del posible cambio climático sobre los recursos hídricos, el diseño y la planificación hidrológica en la España peninsular". El Campo, 137: 201-222.

BISDOM, E.B.A., DEKKER, L.W. \& SCHOUTE, J.F.T. (1993) "Water repellent of sieve fractions from sandy soils and relationships with organic material and soil structure". Geodema, 56: 105-118.

BISHAY, B.G. \& BAKHATI, H.K. (1976) - "Water repellency of soil under citrus tree in Egypt and means of improvement". Agricultural Research Review, 54: 63-74.

BURCH, G.J., MOORE, I.D. \& BURNS, J. (1989) - "Soil hydrophobic effects on infiltration and catchment runoff". Hydrological Processes, 3: 211-222.

CAPRIEL, P. (1997) - "Hydrophocity of organic matter in arable soils: influence of management". European Journal of Soil Science, 48: 457-462.

CEBAllos, A., FERREIRA, A.J.D., COELHO, C.O.A. \& BOULET, A.K. (1999) - "Análisis de la repelencia al agua en una pequeña cuenca hidrográfica afectada por fuego controlado en el área montañosa central de Portugal". Pirineos, 153-154: 123-143.

CEBALLOS, A.; MARTÍNEZ-FERNÁNDEZ, J., SANTOS, F. \& ALONSO, P. (2002a) - "Soil-water behaviour of sandy soils under semi-arid conditions in the Duero Basin". Journal of Arid Environments, 51: 501-519. 
CEBALLOS, A.; CERDÀ, A. \& SCHNABEL, S. (2002b) - "Runoff Production and Erosion Processes on a Dehesa in Western Spain, a Field Rainfall Simulation Approach". Geographical Review, 92: 333-353.

CEBALlos, A., MARTÍNEZ FERNÁNDEZ, J., YUSTE, C. \& LUENGO UGIDOS (2002c) - "Comportamiento hidrológico de suelos texturalmente contrastados del sector central de la Cuenca del Duer"o. En Pérez González, A., Vegas, J. \& Machado, M.J. (eds): Aportaciones de la Geomorfología de España en el inicio del Tercer Milenio:241-248. Ministerio de Ciencia y Tecnología, Instituto Geológico y Minero de España. Madrid.

CEBALLOS, A.; MARTÍNEZ-FERNÁNDEZ, J. \& LUENGOUGIDOS, M.A. (2004) - "Analysis of rainfall trends and dry periods on a pluviometric gradient representative of Mediterranean climate in the Duero Basin", Spain. Journal of Arid Environments, 58: 215-233.

CERDÀ, A., SCHNABEL, S., CEBALLOS, A. \& GÓMEZ AMELIA, D. (1998) - "Soil hydrological response under simulated rainfall in the Dehesa Ecosystem, Extremadura, SW Spain". Earth Surface Processes and Landforms, 23: 195-209.

CHAKRABARTI, D.C. (1971) - "Investigation on erodibility and water stable aggregates of certain soils of Eastern Nepal". Indian Society of Soil Science Journal, 19: 441-446.

CONTRERAS, S. \& SOLÉ, A. (2003) - "Hidrofobia en suelos mediterráneos semiáridos: implicaciones hidrológicas para una pequeña cuenca experimental en el SE ibérico". Cuaternario y Geomorfología, 17 (1-2): 29-45.

CROCKFORD, H., TOPADILIS, S. \& RICHARDSON,D.P. (1991) - "Water repellency in a dry sclerophyll cucalypt forest -measuments and processes-". Hydrological Processes, 5: 405-420.

DAS, D.K. \& DAS, B. (1972) - "Characterization of water repellency in Indian soils". Indian Society of Soil Science Journal, 42: $1099-1112$.

DeBANO, L.F. (1981) - "Water Repellent Soils: a state-of-the-art". General Technical Report PSW-46. Pacific Southwest Forest and Range Experiment Station. $21 \mathrm{pp}$.

DeBANO, L.F. (1991) - "The effects of fire on soil properties". USDA, Forest Service General Technical Report INT-280: 151-156.

DeBANO, L. F. (2000) - "Water repellency in soils: a historical overview". Journal of Hydrology, 231-232: 4-32.

DeBANO, L.F. \& LETEY, J. (Eds) (1969) - "Proceedings of the symposium on water-repellents soils". University of California, Riverside, May 6-10, 1968.

DeBANO, L.F., SAVAGE, S.M. \& HAMILTON, A.D. (1976) "The transfer of heat and hydrophobic substances during burning". Proceedings of the Soil Science Society of America, 40: $779-782$.

DeBANO, L.F., NEARY, D.G. \& FFOLLIOTT, P.F.(1998) - Fire's effects on ecosystem. Wiley, New York. 333 pp.
DEKKER, L.W. (1998) - "Moisture variability resulting from water repellence in Dutch soils. Doctoral Thesis, Wageningen Agricultural University, The Netherlands. $240 \mathrm{pp}$.

DEKKER, L. W. \& RITSEMA, C. J. (1996) - "Preferential flow paths in a water repellent clay soil with grass cover". Water Resources Research, 32: 1239-1294.

DOERR, S.H. (1998) - "On standardizing the "water drop penetration time" and the "molarity of an ethanol droplet" techniques to classify soil hydrophobicity: a case study using medium textured soils". Earth Surface Processes and Landforms, 23: 663-668.

DOERR, S.H., SHAKESBY, R.A. \& WALSH, R.P.D.(1996) - "Soil hydrophobicity variations with depth and particle size fraction in burned and unburned Eucalyptus globulus and Pimuspinaster forest terrain in the Águeda Basin, Portugal". Catena, 27: $25-47$.

DOERR, S.H., SHAKESBY, R.A. \& WALSH, R.P.D. (1998) "Spatial variability of soil hydrophobocity in fire-prone eucalyptus and pine forest, Portugal". Soil Science, 163-4: 313-324.

DOERR, S.H., SHAKESBY, R.A.\& WALSH, R.P.D.(2000) - "Soil water repellency: its causes, characteristics and hydrogeomorphological significance". Earth-Science Reviews, 51: 33-65.

DOERR, S.H.\& THOMAS, A. D. (2000) - "The role of soil moisture in controlling water repellency: new evidence from forest soils in Portugal". Journal of Hydrology, 231-232: 134-147.

EFFIS (2003) - "The European Forest Fires Information System (EFFIS) results on the fire season in Spain up to September $3^{\text {rd }}$, 2003". Update to the report "The European Forest Fires Information System (EFFIS) results on the 2003 fire season in Portugal" (Sept. 15, 2003). .

EMERSON, W.W.(1993) - "Comments of development of seasonal water repellence under direct drilling". Soil Science of America Journal, 57: 877 .

FERREIRA, A.J.D.(1996)-Processos hidrológicos e hidroquímicos em povoamentos de Eucalypius globulus Labill. e Pinus pinaster Aiton. Unpublished Ph.D. thesis, Departamento de Ambiente e Ordenamento, Universidade de Aveiro, Portugal.

FERREIRA, A.J.D., CEBALLOS, A. \& COELHO, C.O.A. (1998) - "Comportamiento hidrófobo del suelo en el oeste peninsular. Metodología y primeros resultados". En Gómez Ortiz, A. y Salvador Franch, F. (eds) - "Investigaciones recientes de la Geomorfología española: 521-528. S.E.G., Universitat de Barcelona. Barcelona.

FERREIRA, A.J.D.; COELHO, C.O.A.; WALSH, R.P.D.; SHAKESBY, R.A.; CEBALLOS, A. \& DOERR, S.H. (2000) - "Hydrological implications of soil water repellency in Eucaliptus globulus forest, North-Central Portugal". Journal of Hydrology, 231-232: 165-177.

FRANCO, C.M.M., TATE, M.E. \& OADES, J.M. (1994) - "The development of water repellency in sands: studies on the physico-chemical and biological mechanisms". Proceedings 
of the Second National Water Repellency Workshop, Perth, Westerm Australia: 18-30.

GERKE, H.H., HANGEN, E., SCHAAF, W. \& HÜTTL, R. F. (2001) - "Spatial variability of potential water repellency in a lignitic mine soil afforested with Pinus nigra". Geoderma, 102: $255-274$

GIOVANNINI, G., LUCCHESI, S. \& GIACHETTI, M. (1987) "The natural evolution of a burned soil: a three-year investigation". Soil Science, 143-3: 220-226.

GRELEWICZ, A. \& PLICHTA, W. (1985) - "The effect of the physical state of the surface of organic soil material on its wettability". Forest Ecology and Mangement, 11: 245-256.

HARPER, R.J. \& GILKES, R.J. (1994) - "Soil attributes related to water-repellency and the utility of soil survey for predicting its occurrence". Australian Joumal of Soil Research, 32: 1109-1124.

HENDRICX, J.M.H., DEKKER, L.W. \& BOERSMA, O.H. (1993) - "Unstable wetting fronts in water repellent field soils". Journal of Environmental Quality, 22: 109-118.

HOLZHEY, C. S. (1969) - "Water-repellent soils in southern California". En: Proceedings Symposium Water-Repellent Soils: 31-42. 6-10 May 1968, University of California, Riverside.

HOUSE, M.G. (1991) - Select Committee Enquiry in Land Conservation Legislative Assembly, Perth, Western Australia.

IMESON, A.C., VERSTRATEN, J.M., VAN MULLINGEN, E.J. \& SEVINK. J. (1992) - "The effects of fire and water repellency on infiltration and runoff under Mediterranean type forests". Catena, 19: 345-361.

INGELMO, F., MOLINA, M.J., RAMOS, M. \& RUIZ M. (2000) "Testing and measuring water repellency in Mediterranean soils". Abstract Book of the Third International Congress of European Society for Soil Conservation: 200. Valencia.

IPCC (2001) - Climatic Change 2001: The Scientific Bases. Contribution of Working Group I to the Third Assessment Report of the Intergovernmental Panel on Climate Change. Houghton, J.T., Ding, Y., Griggs, D.J., Noguer, M., van der Linden, P.J., Dai, X., Maskell, K. \& Johnson, C.A. (eds). Cambridge University Press: United Kingdom. 881 pp.

JAMISON, V. C. (1947) - "Resistance of wetting in the surface of sandy soils under citrus trees in central Florida and its effect upon penetration and the efficiency of irrigation". Soil Science Society of America Proceedings, 11: 103-109.

JARAMILLO,D.F., DEKKER,L.W., RITSEMA, C.J.\&HENDRICX, J.M.H. (2000) - "Ocurrence of soil water repellency in arid and humid climates". Journal of Hydrology, 231-232: 105-111.

JOHN, P.H. (1978) - "Heat-induced water repellency in some New Zealand and pumice soils". New Zealand Joumal of Science, 21: 401-407.

JUNGERIUS, P.D. \& DE JONG. J.H. (1989) - "Variability of water repellency in the dunes along the Dutch coast". Catena, 16: 491-497.
MALLIK, A. U. \& RAHMANN, A. A. (1985) - "Soil water repellency in regularly burned Calluna heatlands: comparison of three measuring techniques". Environmental Management, 20: 207-218.

MARTÍN-VIDE, J. \& GÓMEZ, L. (1999) - "Regionalization of peninsular Spain based on the length of dry spells". International Journal of Climatology, 19: 537-555.

MATAIX-SOLERA, J. \& DOERR, S.H. (2002) - "Hydrophobicity and aggregate stability in calcareous topsoils from fire-affected pine forests in southeastern Spain". Geoderma, 118: 77-88.

McGUIE, D.A. \& POSNER, A.M. (1980) - "Water repelence of a heavy-textured western Australia surface soil". Australian Joumal of Soil Research, 27: 797-806.

McKISSOCK, I., GILKES, R.J., HARPER, R.J. \& CARTER, D.J. (1998) - "Relationships of water repellency to soil properties for different spatial scales of study". Australian Journal of Soil Research, 36: 495-507.

MEEUWIG, R.O. (1971) - "Infiltration and water repellency in granitic soils". USDA Forest Service, Research PaperINT-111.

MOORE, G. \& BLACKWELL, P. (1998) - "Water repellence". En Moore, G. (Eds) - Soil Guide, a handbook for managing agricultural soils. Agriculture Westerm Australian Bulletin 4343: 53-63.

MORAL, F.J., LAGUNA, A. \& GIRÁLDEZ, J.V. (2001) "Generación de escorrentía en los suelos hidrófobos de la zona dunar del SO de la Península Ibérica". En J. J. López y M. Quemada (eds) - Temas de Investigación en Zonas no Saturada: 139-143 Universidad Pública de Navarra. Pamplona.

MORAL, F.J., LAGUNA, A. \& GIRÁLDEZ, J.V. (2002) - "La hidrofobia en los suelos arenosos del parque natural de Doñana: caracterización y distribución". Ingeniería del Agua, 9: $37-50$.

NAKAYA, N., MOTOMURA, S. \& YOKOI, H. (1977) - "Some aspects of water repellency in soils". Soil Science and Plant Nutrition, 23: 409-415.

PRESCOTT, J. A. \& PIPER, C. S. (1932) - "The soils in the South Australian mallee". Trans. R. Soc. S. Aust., 56: 118 pp.

REBELO, F. (2003) - Riscos Naturais e Acção Antrópica. Estudos e reffexões. Imprensa da Universidade, Coimbra. $286 \mathrm{pp}$.

REEDER, C. J. \& JUERGENSEN, M. F. (1979) - "Fire-induced water repellence in forest soils of upper Michigan". Canadian Journal of Forest Research, 9: 369-373.

RICHARDSON, J.L. \& HOLE, F.D. (1978) - "Influence of vegetation on water-repellency in selected western Wisconsin soils". Joumal of the Soil Science Society of America, 42: 465-467.

RIETVELD, J. (1978) - Soil non-wettability and its relevance as a contributing factor to surface runoff on sandy soils in Mali. An ingenieurs research. Landbouw Hogeschool, Wageningen. $179 \mathrm{pp}$. 
RITSEMA, C.J. (1998) - Flow and transport in water repellent sandy soils. Doctoral Thesis. Wageningen Agricultural University, The Netherlands. $215 \mathrm{pp}$.

ROBERTS, F.J. \& CARBON, B.A. (1971) - "Water repellence in sandy soils of south-western Australia. 1. Some studies related to fiel occurrence". Division of Plant Industry CSIRO (Australia), Field Station Record, 10: 13-20.

SCHOLL, D.B. (1975) - "Soil wettability and fire in Arizona chaparral". Soil Science Society of America Proceedings, 39: 356-361.

SCOTT, D.F. (1992) - "The influence of vegetation type on soil wettability". Proceedings of the XVII Congress of the Soil Science Society of South Africa: 10B21-10B26. University of Stellenbosch.

SCOTT, D.F. (1993) - "The hydrological effects of fire in South African mountain catchments". Journal of Hydrology, 150: 409-432.

SCOTT, D. F. (2000) - "Soil wettability in forested cachtments in South Africa, as measured by different methods as affected by vegetation and soil characteristics". Journal of Hydrology, 231-232: 87-104.

SCOTT, D.F., VERSFELD, D.B. \& LESCH, W. (1998) - "Erosion and sediment yield in relation to afforestation and fire in the mountains of the Western Cape Province, South Africa". South African Geographical Journal, 80-1: 52-59.

SCOTT, D. F. \& VAN WYK, D. B. (1990) - "The effects of wildfire on soil wettability and hydrological behaviour of an afforested catchment". Journal of Hydrology, 121: 239-256.

SEVINK, J., IMESON A.C. \& VERSTRATEN, J.M. (1989) "Humus form development and hillslope runoff, and the effects of fire and management, under Mediterranean forest in NE Spain". Catena, 16: 461-475.

SHAKESBY, R.A., COELHO, C.O.A., FERREIRA, A.J.D., TERRY J.P. \& WALSH. R.P.D. (1993) - "Wildfire impacts on soil erosion and hydrology in wet Mediterranean forest", Portugal. International Journal of Wildland Fire, 3: 95-110.

SHAKESBY, R. A.., COELHO, C.O.A., FERREIRA, A.J.D., TERRY, J.P. \& WALSH, R.P.D. (1994) - "Fire post-burn land management practice and soil erosion response curves in eucalyptus and pine forest, North-Central Portugal". En Sala, M. y Rubio, J.L. (Eds): Soil erosion and degradation as a consequence offorest fires: $15-27$. GeoformaEdiciones. Logroño.
SHAKESBY, R.A., BOAKES, D.J., COELHO, C.O.A., GONÇALVES, A.J.B. \& WALSH, R.P.D. (1996) - "Limiting the soil degrational impacts of wildfire in pine and eucalyptus forests, Portugal: a comparison of alternative post-fire management practices". Applied Geography, 16: 337-355.

SHAKESBY, R.A., DOERR, S.H. \& WALSH, R.P.D.(2000) - "The erosional impact of soil hydrophobicity: current problems and future research directions". Journal of Hydrology, 231-232: 178-191.

SOTO, B., BASANTA, R., BENITO, E., PÉREZ, R. \& DİAZ FIERROS (1994) - "Runoff and erosion from burnt soils in northwest Portugal". En Sala, M. y Rubio, J.L. (Eds) - Soil erosion and degradation as a consequence offorest fires: 91-98. Geoforma Ediciones. Logroño.

TAYLOR, D.H. \& BLAKE, G.R. (1982) - "The effect of turfgrass thatch on water infiltration rates". Soil Science Society of America Journal, 46: 616-619.

WALLIS, M.G., HORNE, D.J. \& MCAULIFFE, K.W. (1990) - "A study of water repellency and its amelioration in a yellow brown sand: 1 . Severity of water repellency and the effects of wetting and abrasion". New Zealand Joumal of Agriculture Research, 33: 139-144.

WALLIS, M.G. \& HORNE, D.J. (1992) - "Soil water repellency". Advances in Soil Science, 20: 91-146.

WAL.LIS, M.G., HORNE, D.J. \& PALMER, A.S. (1993) - "Water repellency in a New Zealand development sequence of yellow-brown sands". Australian Joumal of Soil Research, 31: 641-645.

WALSH, R.P.D., BOAKES, D., COELHO,C.O.A., GONÇALVES, A.J.B., SHAKESBY, R.A. \& THOMAS, A.D.(1994) - "Impact of fire-induced hydrophobocity and post-fire forest litter on overland flow in nothern and central Portugal". Proceedings of the Second International Conference on Forest Fire Research, vol. II: 1149-1159. Coimbra, Portugal.

WILKINSON, J.E. \& MILLER, R.H. (1978) - "Investigation and treatment of localized dry spots on sand golf greens". Agronomy Journal, 66: 299-304.

WILSON, G.V.: JARDINE, P.M., LUXMOORE, R.J. \& JONES, R.J. (1990) - "Hydrology of a forested hillslope during storm events". Geoderma, 46: 119-138.

YORK, C. (1998) - "Water repellent soils as they occur on UK golf greens". International Workshop on Soil Water Repellency: origins, assesment, occurrence, consequences, modelling and amelioration: 18. Wageningen, The Netherlands. 